\title{
EDITORIAL
}

\section{New strategies for massive transfusion}

Treatment of anaemia has changed substantially since the early 1990s. In trauma, a subset of patients will require a significant volume of blood. Recent military experience has shown the value of "damage control resuscitation". This implies that damage control techniques are used from the time of injury, by minimising the time between injury and care, and providing aggressive control of haemorrhage, prevention of hypothermia and acidosis, optimising haemodynamic management in intensive care units, and rationalising transfusion support in severely injured patients. The military use of whole blood has minimised some of the risks of component therapy, and has also shown that survival is improved. From this philosophy has come the change in protocol in civilian practice towards early haemostasis, limiting the use of crystalloid or fluid resuscitation (hypotensive resuscitation), and the early use of blood and blood products to maintain the normal coagulation profile as much as possible. Civilian surgeons have modified their practice, and the result has been an improvement in the outcomes of these patients.

Despite questions about the safety of massive blood transfusion, there is no reported upper limit to the utility of additional numbers of red cell units during massive transfusion. Massive transfusion can be lifesaving for the severely injured, however there are an increasing number of studies questioning the long term consequences and complications of early massive transfusion for trauma, and especially the coagulopathy which can result. This coagulopathy has many origins, including dilution, interference with clotting factors and the clotting cascade, temperature, and in the older patient may include concomitant pre-existing use of anticoagulants such as warfarin for cardiac arrhythmias.

Blood cell transfusions have been shown to have significant immunosuppressive potential, and additionally, transmission of fatal diseases through the blood supply has been extensively documented. Although the blood-banking community has systematically reduced the risk of transmission of infection by restricting the eligible donor pool and routinely testing blood products for serologic and nucleic acid evidence of pathogens, comparatively little progress has been made in defining and controlling the immune-modulatory effects of transfusion, and work has shown an increase in sepsis related to the units of blood transfused. Moreover, in at least one large trial, the Transfusion Requirements in Critical Care (TRICC) study, found that using a restrictive RBC transfusion protocol (aimed at maintaining a haemoglobin concentration of 7.0 to $9.0 \mathrm{~g} / \mathrm{dL}$ ) in place of a more traditional protocol (aimed at maintaining a haemoglobin concentration of 10.0 to $12.0 \mathrm{~g} / \mathrm{dL}$ ) did not harm critically ill patients and even appeared to improve survival for younger or less severely ill patients. The volume of blood transfusion required is reduced, thereby minimising the side effects.

However, there will be certain situations where a large amount of blood ( $>2$ units) will need to be administered rapidly, and the concept of a massive transfusion protocol (MTP) has been developed which allows protocol driven infusion of massive amounts of blood in a planned fashion, whilst minimising the time taken for initiation, and the complications arising from the transfusion itself.

Blood Pressure (BP) and Hearst Rate (HR) are the current standard-of-care monitors of shock resuscitation in the initial phase of resuscitation, associated with serum lactate or base excess (base deficit). Both, however, are insensitive markers of early compensated shock; alternative monitors are badly needed for assessing the adequacy of tissue perfusion with a view to avoiding both under-resuscitation and over-resuscitation. Blind and aggressive volume loading in the hope of normalizing BP and HR, without appropriate emphasis on control of haemorrhage, sets the stage for the so-called bloody vicious cycle or the abdominal compartment syndrome Among the most devastating of the complications of massive blood loss and fluid resuscitation is a bleeding diathesis. The coagulopathy of trauma is a syndrome of non-surgical bleeding from mucosal lesions, serosal surfaces, and wound and vascular access sites - the tissue oozing that continues after identifiable vascular bleeding has been controlled. The early addition of plasma and platelets can help the coagulopathy of trauma. If coagulopathy is suspected, the routine use of thrombo-elastography (TEG) as part of goal-directed haemostasis, will assist not only in confirmation of the coagulopathy, but may highlight specific deficiencies in the clotting mechanisms. 
Although massive transfusion may be necessary, trauma surgeons have learnt that this is not their primary objective in the management of the bleeding patient. The primary objective has been to understand and restore the physiology of the trauma patient as quickly as possible, or at least prevent further deterioration. Together with a directed massive transfusion (MTP), this philosophy has the potential to save many lives.

\section{K.D. Boffard,}

BSc(Hons), MBBCh, FRCS, FRCS(Ed) FRCPS(Glas), FACS, FCS(SA), FCSSL(Hon)

Professor Emeritus

Trauma Director Milpark Hospital

Department of Surgery

University of the Witwatersrand

South Africa 